\title{
Numerical modelling of discrete gas flow pathways in water saturated clay
}

\author{
Joaquín Liaudat ${ }^{1}$, Anne-Catherine Dieudonné ${ }^{1}$ and Philip J. Vardon ${ }^{1}$ \\ ${ }^{1}$ Delft University of Technology, Department of Geoscience and Engineering, Stevinweg 1, 2628 CN Delft, The Netherlands
}

\section{Introduction}

Geological Disposal Facilities (GDF) for radioactive waste will generally rely on clay-rich materials as a host geological formation and/or engineered barrier. Gas will be produced within the GDF, which can build up significant gas pressure and activate the migration of gas through the clay materials. Four different transport mechanisms are distinguished based on phenomenological considerations: i) advection and diffusion of gas dissolved in the pore water, ii) viscocapillary two-phase flow, iii) dilatancy controlled gas flow (pathway dilation), and iv) gas transport along macroscopic tensile fractures (gas fracturing). Although significant research has been carried out on this topic, there are still significant gaps in the understanding of the factors controlling the activation, evolution and stability of mechanisms ii, iii and iv. Along this line, the authors present a numerical model which is being developed with the ambition to reproduce, within a single framework, the four gas transport mechanisms at the scale of laboratory gas injection tests, in order to study the interactions among them, and, in particular, the formation of discrete gas flow pathways.

The model is developed using the Finite Element Method, based on a fully coupled Pneumo-HydroMechanical (PHM) formulation in 2D, and has been implemented in the code LAGAMINE. Two different types of finite elements are used: solid and interface elements (see Figure 1). Solid elements are used to represent the mechanical and flow processes in the bulk (continuum) clay material and interface elements are used to represent natural or induced discontinuities, such as fractures or clay-steel contacts in a testing device. In order to model induced fractures, the model parameters are such that, as long as the interface elements remain closed, they do not have any significant effect on the response of the model. However, when a certain mechanical threshold is reached (e.g. the tensile strength) and the interface element starts to open, localised mechanical and flow processes are triggered. Similar approaches can be found in the literature for modelling coupled fracturing processes in geomaterials (e.g. Liaudat et al., 2020).
For the processes in the bulk material (solid elements) the authors have mostly relied on previous developments, while for the discontinuities a new PHM zero-thickness interface element has been developed and implemented in the code. This new element is of the triple-node type, where the outer nodes have 4 degrees of freedom ( $\mathrm{x}$ and y coordinates, liquid phase pressure $\mathrm{p}_{\mathrm{w}}$, and gas phase pressure $\mathrm{p}_{\mathrm{g}}$ ), while inner nodes have only 2 (liquid and gas phase pressures). The element formulation combines concepts from previous works by Segura and Carol (2004, 2008), who developed two-node HM interface elements with a node-to-node contact discretization, and by Cerfontaine et al. (2015) and Dieudonné et al. (2015), who developed triple-node PHM interface elements with a node-to-segment contact discretization.

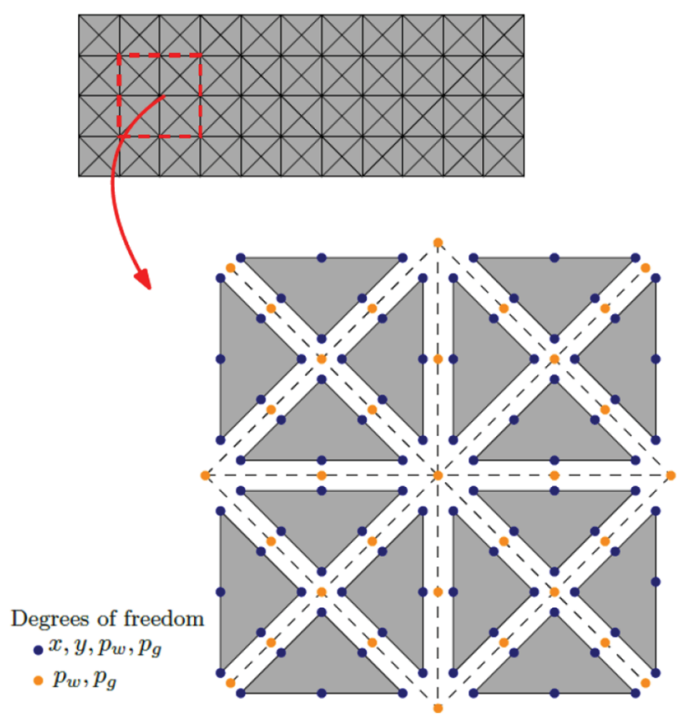

Fig. 1: Introduction of zero-thickness interface elements in a conventional FE mesh.

The flow problem is treated in the typical theoretical framework for two-phase flow in porous medium, considering two chemical species (water and gas) and two fluid phases (liquid and gaseous). The liquid phase includes both liquid water and dissolved gas species,

\footnotetext{
* Corresponding author: j.liaudat@,tudelft.nl
} 
while the gas phase only comprises gas species, i.e. water vapour is not considered. The heterogeneity of the clay transport/retention parameters, which is expected to determine the formation of discrete gas flow pathways, is introduced via the stochastic initialization of selected parameters.

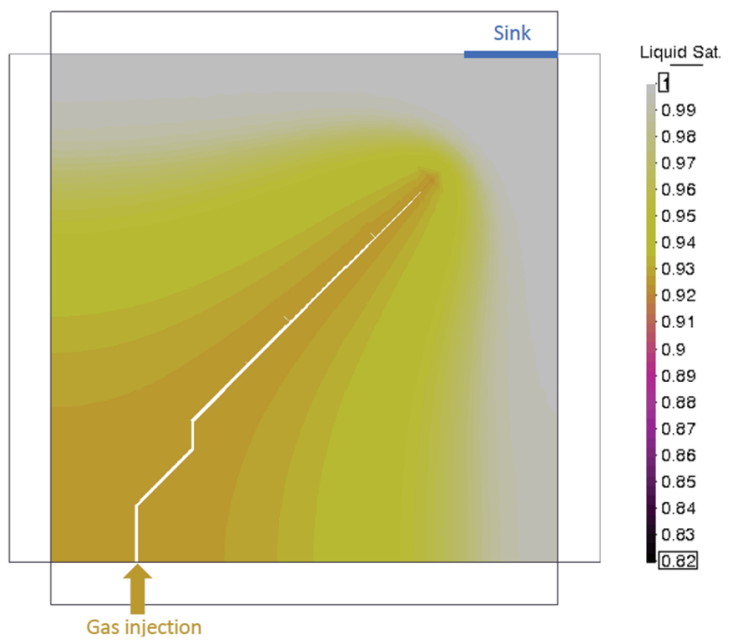

Fig. 2: Gas fracture and liquid saturation degree in a clay sample during an idealised gas injection test. $24 \times 24 \mathrm{~mm}$ Boom clay sample, initial state $\sigma_{x}=\sigma_{y}=4.5 \mathrm{MPa}, p_{w}=2.2 \mathrm{MPa}$.

The mechanical problem is treated with an updated Lagrangian formulation with a node-to-node discretization of the contact area. Typically, the solid elements are assumed to behave linear elastically and nonlinear laws are assigned to the zero-thickness interface elements. However, there is no theoretical restriction to use also a non-linear constitutive law for solid elements.

The coupling between the mechanical and the flow formulations occurs in both directions. On one hand, the fluid phase pressures and the saturation degree are introduced in the mechanical equilibrium equation through the principle of effective stress. On the other hand, the flow properties (gas entry value, storage capacity, longitudinal transmissivity and diffusivity) vary dramatically, governed largely by the mechanical aperture of the discontinuity. To deal with these strong couplings, a monolithic (fully coupled) numerical implementation is used.

In addition to the model formulation, the authors will present preliminary results that show the ability of the model to realistically reproduce observed features of the gas injection tests such as the formation of discrete gas flow pathways (see Figure 2), episodic break-through events and fracture closure (self-sealing) after a breakthrough episode.

\section{Acknowledgements}

This research was performed in the framework of the European Joint Programme on Radioactive Waste Management (EURAD). EURAD has received funding from the European Union's Horizon 2020 research and innovation programme under grant agreement No 847593.

\section{References}

1. Cerfontaine, B., Dieudonné, A. C., Radu, J. P., Collin, F., \& Charlier, R. (2015). "3D zero-thickness coupled interface finite element: Formulation and application." Computers and Geotechnics, 69, 124140.

2. Dieudonné, A. C., Cerfontaine, B., Collin, F., \& Charlier, R. (2015). "Hydromechanical modelling of shaft sealing for CO2 storage." Engineering Geology, 193, 97-105.

3. Liaudat, J., Carol, I., \& López, C. M. (2020). "Model for Alkali-Silica Reaction expansions in concrete using zero-thickness Chemo-Mechanical interface elements". International Journal of Solids and Structures, 207, 145-177.

4. Segura, J. M., \& Carol, I. (2004). "On zero-thickness interface elements for diffusion problems." International Journal for Numerical and Analytical Methods in Geomechanics, 28(9), 947-962.

5. Segura, J. M., \& Carol, I. (2008). "Coupled HM analysis using zero-thickness interface elements with double nodes. Part I: Theoretical model." International Journal for Numerical and Analytical Methods in Geomechanics, 32, 2083-2101. 